\title{
BMJ Open Intervention to reduce benzodiazepine prescriptions in primary care, study protocol of a hybrid type 1 cluster randomised controlled trial: the BENZORED study
}

Caterina Vicens, ${ }^{1,2}$ Alfonso Leiva, ${ }^{2,3}$ Ferran Bejarano, ${ }^{4}$ Ermengol Sempere, ${ }^{5}$
Raquel María Rodríguez-Rincón, ${ }^{6}$ Francisca Fiol, ${ }^{1}$ Marta Mengual, ${ }^{4}$
Asunción Ajenjo, ${ }^{5}$ Fernando Do Pazo, ${ }^{6}$ Catalina Mateu, ${ }^{1,2}$ Silvia Folch, ${ }^{4}$
Santiago Alegret, ${ }^{1}$ Jose Maria Coll, ${ }^{7}$ María Martín-Rabadán, ${ }^{8}$ Isabel Socias ${ }^{2,9}$

\section{ABSTRACT}

Introduction Benzodiazepines (BZDs) are mainly used to treat anxiety and sleep disorders, and are often prescribed for long durations, even though prescription guidelines recommend short-term use due to the risk of dependence, cognitive impairment, and falls and fractures. Education of general practitioners (GPS) regarding the prescription of BZDs may reduce the overuse and of these drugs. The aims of this study are to analyse the effectiveness of an intervention targeted to GPs to reduce BZD prescription and evaluate the implementation process.

Methods and analysis The healthcare centres in three regions of Spain (Balearic Islands, Catalonia and Community of Valencia) will be randomly allocated to receive a multifactorial intervention or usual care (control). GPs in the intervention group will receive a 2-hour workshop about best-practice regarding BZD prescription and BZD deprescribing, monthly feedback about their BZD prescribing practices and access to a support web page. Outcome measures for each GP are the defined daily dosage per 1000 inhabitants per day and the proportion of long-term BZD users at 12 months. Data will be collected from the electronic prescription database of the public health system, and will be subjected to intention-to-treat analysis. Implementation will be evaluated by mixed methods following the five domains of the Consolidated Framework For Implementation Research.

Ethics and dissemination This study was approved by the Balearic Islands Ethical Committee of Clinical Research (IB3065/15), I'IDIAP Jordi Gol Ethical Committee of Clinical Research (PI 15/0148) and Valencia Primary Care Ethical Committee of Clinical Research (P16/024). The results will be submitted for publication in peer-reviewed journals. Trial registration number ISRCTN28272199.

\section{INTRODUCTION}

Benzodiazepines (BZDs) are mainly used to treat anxiety and sleep disorders, but also as an add-on therapy for patients with schizophrenia, depression and alcohol withdrawal.
Strengths and limitations of this study

- To the authors' best knowledge, this is the largest multicentre randomised controlled trial to analyse the effectiveness and implementation of an intervention on benzodiazepine prescription practices among general practitioners (GPS).

- The study will include healthcare practices working in real-world conditions that are representative of the target population for whom the intervention is intended. However, it is possible that GPs from practices that participate might be more motivated to change their practices than those who refuse participation.

- We recruited the healthcare centres before randomisation to avoid possible selection bias.

- Intracluster contamination between healthcare centres may occur within the same organisation, however, potential contamination biases result towards the null hypothesis.

- Outcome assessment will be blinded to the control and intervention arms. Blinding of participants and healthcare professionals is not possible due to the nature of the intervention.

These drugs also have anticonvulsant properties and are muscle relaxants. ${ }^{1}$

Prescription guidelines recommend shortterm use of BZDs, no longer than 4 weeks for insomnia ${ }^{2}$ and $4-8$ weeks for anxiety, ${ }^{3}$ because long-term use can lead to tolerance and dependence, and other adverse effects such as somnolence, daytime drowsiness, cognitive decline, ${ }^{4-6}$ falls resulting in hip fracture $^{7-9}$ and motor vehicle accidents. ${ }^{10-12}$ Moreover, some recent studies found an association of regular use of sedative drugs with increased mortality. ${ }^{13-15}$ Despite these recommendations, many studies reported 
long-term use and overprescription of BZDs, particularly in older people. ${ }^{16}{ }^{17}$ In contrast to the declines in BZD use in other European countries during the last decade, BZD use continues to increase in Spain. ${ }^{18} 19$

Most BZDs are prescribed in primary care by general practitioners (GPs), and the prevalence of their use varies enormously among countries, regions and healthcare settings. ${ }^{20}$ BZD use depends on several factors, ${ }^{21}$ including sociodemographical characteristics of the patient (age, sex and comorbidities), the prescribing habits of the physician, the expectation and perceptions of risks and benefits, and knowledge of alternative approaches. ${ }^{22}$

The recommended clinical regimen for deprescribing BZD is gradual tapering. ${ }^{1}$ Several studies of discontinuation programmes and meta-analysis have shown this approach is effective in reducing long-term BZD consumption, ${ }^{23-29}$ some of these approaches are under the guidance of a GP. ${ }^{23} 243031$

Many medical interventions shown to be effective in research studies fail to translate into significant health outcomes, because of barriers to the dissemination of the evidence in routine clinical practice. ${ }^{31}$

Educational face-to-face meetings and workshops, as well as audit/feedback targeted to GPs, have been widely used in clinical practices to improve healthcare outcomes. ${ }^{32} 33$ These approaches provide essential knowledge and practical algorithms from updated treatment guidelines, and are considered effective methods for improving professional practice and prescribing habits. ${ }^{34}$ These approaches may also be useful for reducing the prescription of BZDs. ${ }^{35-37}$

We aim to analyse the effectiveness of an intervention consisting of an educational training workshop to GPs about the appropriate BZDs prescribing and strategies for successful deprescribing, monthly feedback about their BZD prescription and access to a support web page, to decrease the BZD prescription and the proportion of long-term BZD users. We also aim to analyse the implementation process of the intervention following the five domains of the Consolidated Framework For Implementation Research (CFIR) ${ }^{31}$

\section{OBJECTIVES}

\section{Main objective}

To evaluate the effectiveness of a primary care intervention in which GPs are given training in the initial prescription of BZDs and BZD deprescribing process for long-term users, monthly feedback on BZD prescriptions and access to a support web page, to reduce a BZD prescriptions measured by the defined daily dosage per 1000 inhabitants per day (DHD). A reduction of one DHD is interpreted as on average one patient in a representative group of 1000 inhabitants would have ceased from taking a daily standard dose of BZD, during the year analysed.

\section{Secondary objectives}

To evaluate the effectiveness of the intervention on the proportion of BZD long-term users at 12 months:

- Total population.

- Individuals $\geq 65$ years old.

To analyse the implementation process following the five domains of the CFIR.

\section{METHODS AND ANALYSIS \\ Study design and setting}

A type 1 hybrid effectiveness-implementation two-arm cluster randomised clinical trial in a primary care setting will be performed in three regions of Spain: Balearic Islands (IbSalut), Catalonia (Institut Català de la Salut;Tarragona-Reus district) and Community of Valencia (Conselleria de Salut Universal; Arnau de Vilanova llíria district). The cluster design was selected because the allocation and intervention will be implemented at the level of the healthcare centre.

\section{Eligibility criteria and recruitment}

All primary care healthcare centres from the Balearic Islands and the participating health districts of Catalonia and Community of Valencia will be eligible. A researcher will contact the head of each healthcare centre and explain the nature of the project and invite participation.

Inclusion criteria: Healthcare centres with acceptance from at least two-thirds of GPs to participate in the study.

\section{Sample size}

Sample size calculation was based on a reduction between groups of at least five BZDs DHD. The DHD is defined as the number of prescribed WHO's defined daily doses (WHO's DDDs)/1000 inhabitants/year. Mean DHD of BZD prescription in the participating regions was 75 and an SD of 18.7 DHD. This leads to our initial estimate that 220 GPs are needed in the intervention arm and 220 GPs in the control arm.

We also corrected for correlation among GPs within clusters using the inflation factor $1+\mathrm{p}(\mathrm{m}-1),{ }^{38}$ where $\mathrm{m}$ is the mean number of observations per cluster and $p$ is the intraclass correlation coefficient. Each participating healthcare centre will include approximately 10 GPs, the expected intraclass correlation coefficient between clusters is $0.05^{39}$ and the cluster design effect is 1.45 . Thus, the final target sample size is 638 GPs (319 in each arm).

\section{Random allocation}

Randomisation will be stratified according to the DHD of the healthcare centre, proportion of patients older than 65 years and geographical region. Healthcare centres will be included if two-thirds of the GPs accept participation.

Concealment of allocation: After all eligible healthcare centres accept participation the coordinating centre will allocate them simultaneously to intervention or control group using a computer-generated random number tables. 
BenzoRed

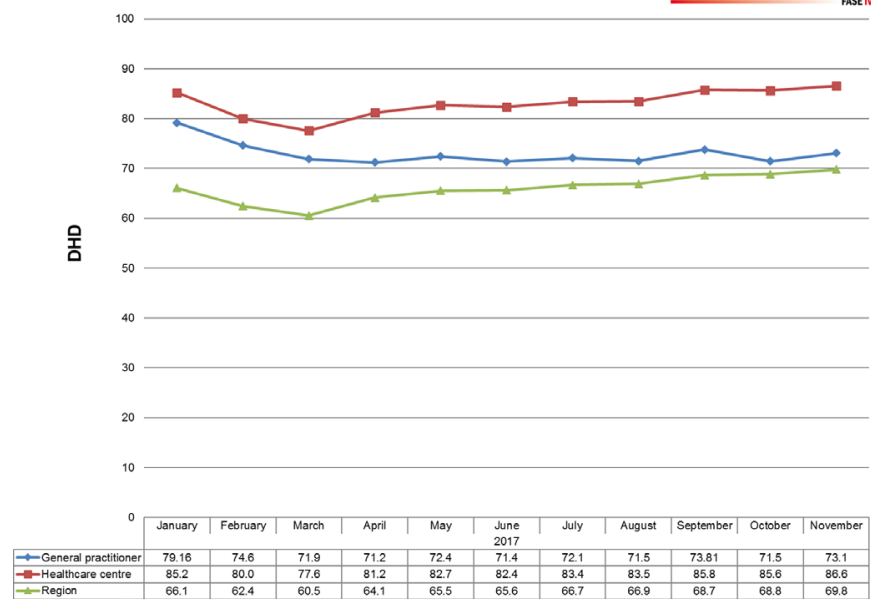

Figure 1 Monthly information received by each general practitioner (GP) over 12 months . GP average

DHD compared with the average of the healthcare centre and the region. DHD, defined daily dosage per 1000 inhabitants per day.

\section{Blinding}

GPs cannot be blinded to the intervention that seeks to modify their clinical practice, nor can the researchers be blinded to the allocation of the GPs. However, the researchers and the data analyst will be blinded to the final outcomes. The primary and secondary outcomes will be assessed by examination of the electronic prescription database for each GP. These data will be extracted and anonymised by an external data manager who is not involved in the study and is blinded to patient allocation.

\section{Intervention}

The multifactorial intervention has three main components:

1. Educational workshop training: GPs in the intervention group centres will attend 2-hour workshops in their workplaces. The content of the workshop will include:

A. Rationale for prescribing BZDs: main indications; pharmacological properties (half-life, equivalent doses of the main prescribed agents); prevalence of long-term use; adverse outcomes and how to start a prescription.

B. Strategies for deprescribing long-term BZD use: a patient-structured educational interview and tailored gradual tapering consisting of a $10 \%-$ $25 \%$ reduction in the daily doses every $2-3$ weeks.

2. Audit and feedback: Each month, all participating GPs will be sent information about their BZD prescriptions in DHD, in a monthly data chart over the 12 months of the study (figure 1), and a comparison of their data with the average of the healthcare centre and the region. At 0,6 and 12 months, GPs will also receive a chart with their prescription in DHD compared with the GPs of the healthcare centre (figure 2).

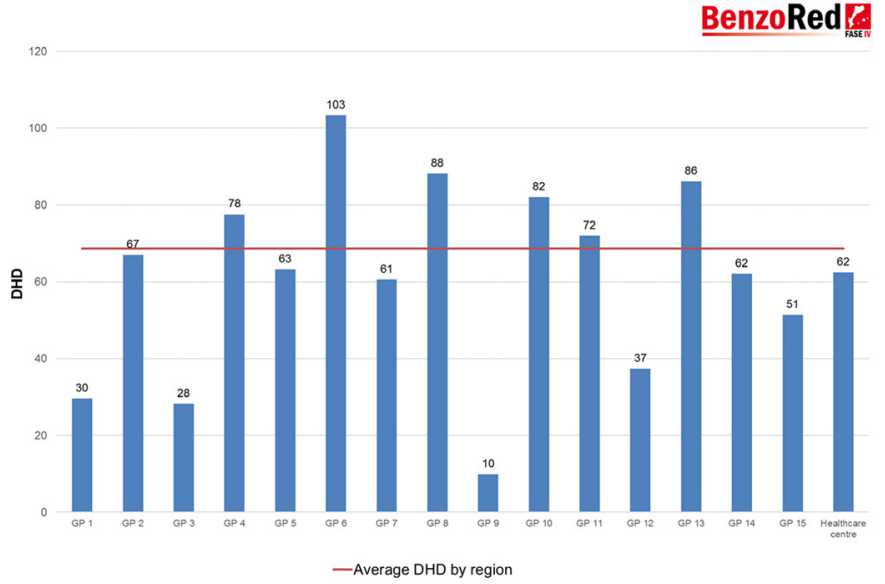

Figure 2 Comparative BZD prescription between GPs of the healthcare centre. BZD, benzodiazepine; DHD, defined daily dosage per 1000 inhabitants per day; GPs, general practitioners.

3. Training and support web page (figure 3): GPs will be given general information on BZDs, the rationales for BZD use and effective strategies to reduce BZD use. This will consist of a 20 min video that summarises the main aspects of the intervention, support information for GPs about adverse effects of BZD, equivalent doses of the different types of BZDs, a self-help leaflet for patients to improve sleep quality, and written recommendations that reinforce the educational information (risk and benefits, tolerance, dependence and clinical problems related to long-term use) provided by the GPs.

Attending the workshop training, downloads of the monthly feedback reports and visits to the support web page will be registered and monitored. Healthcare centres randomised to the usual care group will not

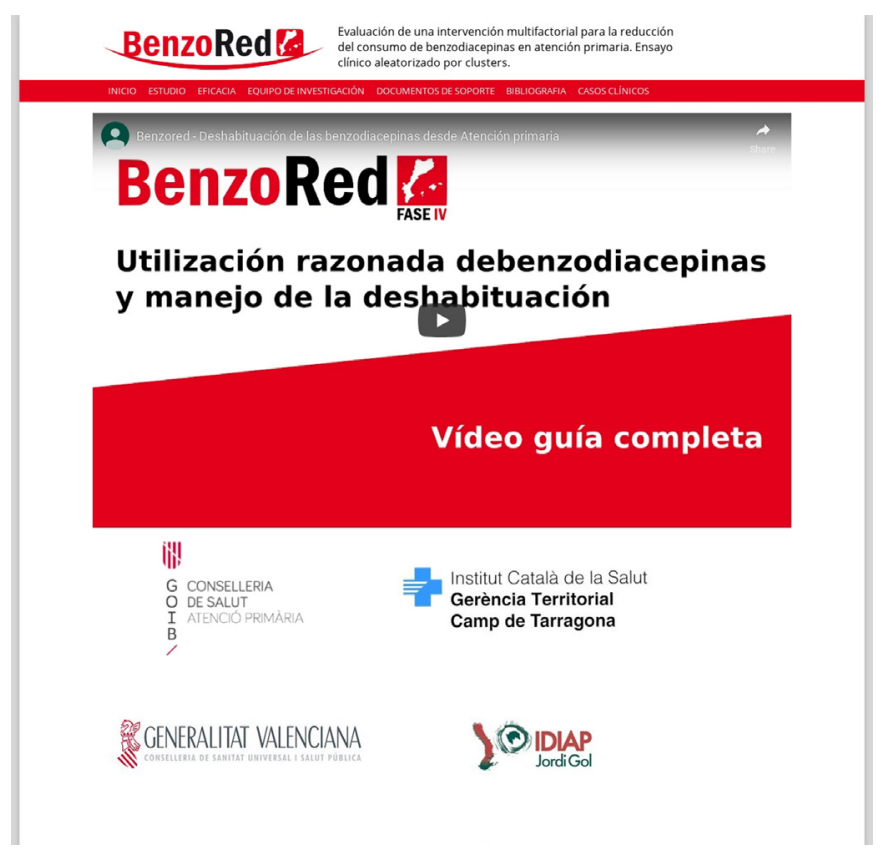

Figure 3 BenzoRed support web page for professionals. 
receive any component of these interventions (training, feedback and access to the support web page).

\section{Outcome assessment}

Primary and secondary outcomes will be extracted from the electronic prescription drug claims database of the public health system of each region, under supervision of the pharmacist researchers of the study.

\section{Primary outcome measure}

The primary outcome measure is the GPs' DHD of BZDs at 12 months after the training workshop. We defined DHD as the number of DDD/1000 inhabitants/year. The definition of DDD by WHO is the 'assumed average maintenance dose per day for a drug used for its main indication in adults'. The included BZDs are all agents in the following Anatomical Therapeutic Chemical classification system-coded groups: N05BA, N05CD and N05CF.

\section{Secondary outcome measures \\ Effectiveness}

Proportion of long-term ( $>6$ months) BZD users at 12 months.

Proportion of long-term ( $>6$ months) BZD users aged 65 or more at 12 months.

We defined long-term use as daily use of a BZD for at least 6 months. GPs who decline to participate or left practice during the follow-up period will be asked to provide consent for analysis of their data at 12 months.

\section{Implementation}

The CFIR describes 39 constructs across five domains that systematically assess and articulate contextual factors that may influence the implementation process. The five major domains are: intervention characteristics, outer setting, inner setting, characteristics of the individuals involved and the process of implementation.

To explore all the constructs of the five domains of the CFIR, we will use a mixed methods approach.

\section{Quantitative data}

We designed a standardised form including 41 general and specific questions 10-point Likert scale type to assess the 39 CFIR constructs.

\section{Qualitative data}

Five focus groups of participants GPs will address all CFIR constructs to determine facilitators and barriers to the implementation. The focus groups will be conducted at the practices and will last about $90 \mathrm{~min}$. They will be digitally recorded and transcribed verbatim by trained transcriptionists.

\section{Patient and public involvement}

Previous to the clinical trial, long-term BZDs users were invited to participate in a focus group to discuss the aspects they were concerned about BZD use to design a meaningful message targeted to patients, easy to understand with key aspects considered important in order to deprescribe BZD drugs.

About the burden of the intervention, our previous study showed that an intervention with a single visit was as effective as a more complex intervention adding several follow-up visits.

Patients or public were not involved in the design of this trial, the development of the research question or outcome measures.

\section{Data management}

Each GP's BZD prescription data will be coded using a unique numerical identification in a secured and validated electronic database. Logical checks will be performed to identify missing data and inconsistencies. The researcher and data analysts will have full access to these data.

\section{Statistical analysis}

Estimated effects will be calculated by comparing the DHDs of GPs in the intervention and control groups at 12 months. A generalised mixed linear random effect models will be used to account for clustering at the level of the healthcare centre, and adjusted for baseline values of the outcome measures. All analyses will be performed on an 'intention-to-treat' basis (ie, all initially enrolled GPs will be included in the analysis according to the group to which they were assigned) and will be reported according to 2010 Consolidated Standards of Reporting Trials guidelines. Subgroup analysis of effectiveness by regions will also be performed. We will also test for significant differences in the baseline characteristics of the control and intervention groups using descriptive analysis. This will include calculation of means and/or proportions with CIs, and on robusts SDs (to account for clustering). We will test for differences in the implementation success by descriptive analysis of the CFIR constructs. .

\section{Ethics and dissemination}

This study will follow the principles outlined in the Declaration of Helsinki (seventh revision). The results of this study will be disseminated via peer-reviewed publications and conference presentations. All data will be available on request.

\section{Author affiliations}

${ }^{1}$ Son Serra-La Vileta Healthcare Centre, Balearic Health Service Ib-Salut, Palma, Spain

${ }^{2}$ Instituto de Investigación Sanitaria de Baleares (IdISBa), Palma, Spain

${ }^{3}$ Primary Care Reseach Unit, Balearic Health Service lb-Salut, Palma, Spain

${ }^{4}$ DAP Camp de Tarragona, Catalunya Health Services-CatSalut, Tarragona, Spain

${ }^{5}$ Paterna Healthcare Centre, Conselleria de Sanitat Universal, Agència Valenciana de Salut, Valencia, Spain

${ }^{6}$ Pharmacy Department, Hospital Universitari Son Espases, Palma de Mallorca, Spain

${ }^{7}$ Menorca Primary Care Management, Balearic Health Service lb-Salut, Mahón, Spain

${ }^{8}$ Can Misses Healthcare Centre, Balearic Health Service Ib-Salut, Ibiza, Spain

${ }^{9}$ Manacor Healthcare Centre, Balearic Health Service lb-Salut, Manacor, Spain

Acknowledgements We are grateful to participants GPs, heads of the healthcare centres for their help in the project development, to Alberto Parra, primary care 
pharmacist, for his help in managing GPs prescription data in the Arnau de Vilanova-Llíria sanitary district (Valencia) and to the research unit of primary care of Mallorca for their methodological support and advice.

Contributors CV, FB, ES, FDP, RMR-R and AL collectively drafted the study protocol, and sought funding and ethical approval. FDP, RMR-R, CV, ES and FB have access to the GPs' electronic prescription database, and prepared the monthly report to be sent to the GPs. CV, FB and ES are the steering committee, and are responsible for management of the trial. CV, IS, FF, ES, AA, FB, MM-R, SF, CM and SA are responsible for development and implementation of the training workshop in the practices targeted to GPs. CV, IS, FF, ES, JMC, MM-R, AA, SA, FB, MM, SF, CM and AL will develop the content of the support web page for the study, and develop and implemented the questionnaires. All authors have read the draft critically, made contributions and approved the final manuscript. CV is the principal investigator, will have full access to all the data in the study, and takes responsibility for the integrity of the data and the accuracy of the data analysis.

Funding This study was funded by the Ministry of Economy and Competitiveness, Carlos III Institute (www.isciii.es), grant PI15/01480. We also received support from the Health Promotion and Preventive Activities-Primary Health Care Network, sustained by the Ministry of Health ISCIII-RETIC awards RD16/0007/0008, alsosupported with European Union ERDF funds.

Disclaimer The funders had no role in the study design, data collection, management, analysis, interpretation, decision to publish or preparation of the manuscript.

Competing interests None declared.

Patient consent for publication Not required.

Ethics approval This trial was approved by the Primary Care Research Committee, the Balearic Islands Ethical Committee of Clinical Research (IB3065/15), I'IDIAP Jordi Gol Ethical Committee of Clinical Research (PI 15/0148) and Valencia Primary Care Ethical Committee of Clinical Research (P16/024).

Provenance and peer review Not commissioned; externally peer reviewed.

Open access This is an open access article distributed in accordance with the Creative Commons Attribution Non Commercial (CC BY-NC 4.0) license, which permits others to distribute, remix, adapt, build upon this work non-commercially, and license their derivative works on different terms, provided the original work is properly cited, appropriate credit is given, any changes made indicated, and the use is non-commercial. See: http://creativecommons.org/licenses/by-nc/4.0/.

\section{REFERENCES}

1. BNF: Joint Formulary Committee. British National Formulary. 64th edn. London: British Medical Association and Royal Pharmaceutical Society of Great Britain, 2012.

2. National Institute for Clinical Excellence. Guidance on the use of zaleplon, zolpidem and zopiclone for the short-term management of insomnia. 2004. https://www.nice.org.uk/guidance/ta77/chapter/1Guidance (Accessed 9 Jan 2018).

3. NICE. Anxiety disorders Guidance and guidelines. 2014. https:// www.nice.org.uk/guidance/qs53/chapter/Introduction (Accessed 9 Jan 2018).

4. Billioti de Gage S, Moride Y, Ducruet T, et al. Benzodiazepine use and risk of Alzheimer's disease: case-control study. BMJ 2014;349:g5205.

5. Zhong G, Wang $Y$, Zhang $Y$, et al. Association between Benzodiazepine use and Dementia: a meta-analysis. PLoS One 2015;10:e0127836.

6. Picton JD, Marino AB, Nealy KL. Benzodiazepine use and cognitive decline in the elderly. Am J Health Syst Pharm 2018:75:e6-e12.

7. Bakken MS, Engeland A, Engesæter LB, et al. Risk of hip fracture among older people using anxiolytic and hypnotic drugs: a nationwide prospective cohort study. Eur J Clin Pharmacol 2014;70:873-80.

8. Wang PS, Bohn RL, Glynn RJ, et al. Hazardous benzodiazepine regimens in the elderly: effects of half-life, dosage, and duration on risk of hip fracture. Am J Psychiatry 2001;158:892-8.

9. Khong TP, de Vries F, Goldenberg JS, et al. Potential impact of benzodiazepine use on the rate of hip fractures in five large European countries and the United States. Calcif Tissue Int 2012;91:24-31.

10. Smink BE, Egberts AC, Lusthof KJ, et al. The relationship between benzodiazepine use and traffic accidents: a systematic literature review. CNS Drugs 2010;24:639-53.
11. Barbone F, McMahon AD, Davey PG, et al. Association of road-traffic accidents with benzodiazepine use. Lancet 1998;352:1331-6

12. Orriols L, Philip P, Moore N, et al. Benzodiazepine-like hypnotics and the associated risk of road traffic accidents. Clin Pharmacol Ther 2011;89:595-601.

13. Belleville G. Mortality hazard associated with anxiolytic and hypnotic drug use in the National Population Health Survey. Can J Psychiatry 2010;55:558-67.

14. Kripke DF, Langer RD, Kline LE. Hypnotics' association with mortality or cancer: a matched cohort study. BMJ Open 2012;2: 000850.

15. Weich S, Pearce HL, Croft P, et al. Effect of anxiolytic and hypnotic drug prescriptions on mortality hazards: retrospective cohort study. BMJ 2014;348:g1996.

16. Bejarano Romero F, Piñol Moreso JL, Mora Gilabert N, et al. [Increased benzodiazepine use in elderly women attending urban primary health care centers]. Aten Primaria 2008;40:617-21.

17. Ohayon MM, Lader MH. Use of psychotropic medication in the general population of France, Germany, Italy, and the United Kingdom. J Clin Psychiatry 2002;63:817-25.

18. Vicente Sánchez MP, Macías Saint-Gerons D, Fuente Honrubia Cdela, et al. Evolución del uso de medicamentos ansiolíticos e hipnóticos en España durante el período 2000-2011. Revista Española de Salud Pública 2013;87:247-55.

19. Organisation for Economic Co-operation and Development (OECD) statistics. Pharmaceutical consumption. http://stats.oecd.org/ (Accessed 9 Jan 2018).

20. Soyka M. Treatment of Benzodiazepine Dependence. N Engl J Med 2017;376:1147-57.

21. Dell'Osso B, Albert U, Atti AR, et al. Bridging the gap between education and appropriate use of benzodiazepines in psychiatric clinical practice. Neuropsychiatr Dis Treat 2015;11:11.

22. Sirdifield C, Chipchase SY, Owen S, et al. A Systematic Review and Meta-Synthesis of Patients' Experiences and Perceptions of Seeking and Using Benzodiazepines and Z-Drugs: Towards Safer Prescribing. Patient 2017;10:1-15.

23. Cormack MA, Sweeney KG, Hughes-Jones H, et al. Evaluation of an easy, cost-effective strategy for cutting benzodiazepine use in general practice. Br J Gen Pract 1994;44:5-8.

24. Bashir K, King M, Ashworth M. Controlled evaluation of brief intervention by general practitioners to reduce chronic use of benzodiazepines. Br J Gen Pract 1994;44:408-12.

25. Voshaar RC, Couvée JE, van Balkom AJ, et al. Strategies for discontinuing long-term benzodiazepine use: meta-analysis. $\mathrm{Br} \mathrm{J}$ Psychiatry 2006;189:213-20.

26. Baillargeon L, Landreville $\mathrm{P}$, Verreault $\mathrm{R}$, et al. Discontinuation of benzodiazepines among older insomniac adults treated with cognitive-behavioural therapy combined with gradual tapering: a randomized trial. CMAJ 2003;169:1015-20.

27. Morin CM, Bastien C, Guay B, et al. Randomized clinical trial of supervised tapering and cognitive behavior therapy to facilitate benzodiazepine discontinuation in older adults with chronic insomnia. Am J Psychiatry 2004;161:332-42.

28. Parr JM, Kavanagh DJ, Cahill L, et al. Effectiveness of current treatment approaches for benzodiazepine discontinuation: a metaanalysis. Addiction 2009;104:13-24.

29. Vicens C, Fiol F, Llobera J, et al. Withdrawal from long-term benzodiazepine use: randomised trial in family practice. $\mathrm{Br} J$ Gen Pract 2006;56:958-63.

30. Vicens C, Bejarano F, Sempere E, et al. Comparative efficacy of two interventions to discontinue long-term benzodiazepine use: cluster randomised controlled trial in primary care. Br J Psychiatry 2014;204:471-9.

31. Damschroder LJ, Aron DC, Keith RE, et al. Fostering implementation of health services research findings into practice: a consolidated framework for advancing implementation science. Implement Sci 2009:4:50.

32. O'Brien MA, Rogers S, Jamtvedt G, et al. Educational outreach visits: effects on professional practice and health care outcomes. In: O'Brien MA, ed. Cochrane database of systematic reviews. Chichester, UK: John Wiley \& Sons, Ltd, 2007:CD000409.

33. Ivers N, Jamtvedt G, Flottorp S, et al. Audit and feedback: effects on professional practice and healthcare outcomes. In: Ivers N, ed. Cochrane database of systematic reviews. Chichester, UK: John Wiley \& Sons, Ltd, 2012:CD000259.

34. Forsetlund L, Bjørndal A, Rashidian A, et al. Continuing education meetings and workshops: effects on professional practice and health care outcomes. In: Forsetlund L, ed. Cochrane database of systematic reviews. Chichester, UK: John Wiley \& Sons, Ltd, 2009:CD003030. 
35. Smith AJ, Tett SE. Improving the use of benzodiazepines--is it possible? A non-systematic review of interventions tried in the last 20 years. BMC Health Serv Res 2010;10:321.

36. Smith AJ, Tett SE. An intervention to improve benzodiazepine use--a new approach. Fam Pract 2010;27:320-7.

37. Dou C, Rebane J, Bardal S. Interventions to improve benzodiazepine tapering success in the elderly: a systematic review. Aging Ment Health 2018:1-6.
38. Ukoumunne OC, Gulliford MC, Chinn S, et al. Methods for evaluating area-wide and organisation-based interventions in health and health care: a systematic review. Health Technol Assess 1999;3:iii-92.

39. Campbell M, Grimshaw J, Steen N. Sample size calculations for cluster randomised trials. Changing Professional Practice in Europe Group (EU BIOMED II Concerted Action). J Health Serv Res Policy 2000;5:12-16. 